\title{
BMC Clinical Pathology Reviewer Acknowledgment 2015
}

Elaine Zhang

\section{Contributing reviewers}

The editors of BMC Clinical Pathology would like to thank all our reviewers who have contributed to the journal in Volume 15 (2015).

Eshan Affan

Australia

Atif Ahmed

USA

Lara Alessandrini

Italy

Emyr Wyn Benbow

UK

Fiona Blows

UK

Camilla Böckelman

Finland

Annegien Broeks

Netherlands

Paul Cane

UK

Joshua Coleman

USA

Diego O. Croci

Argentina

Julie Cunningham

USA

Henrique De Amorim Almeida

USA

Ricardo Dos Reis

Brazil
Christina Ellervik
Denmark

Isabel Fonseca

Portugal

Andrea Galli

Italy

Jeff Garner

UK

Tomasz Gosiewski

Poland

Gaurav Gupta

USA

Sultan Habeebu

USA

Rui Henrique

Portugal

Alyson Hunter

Ireland

Jing Jiang

China

Ekaterina Jordanova

Netherlands

Kevin Kalinsky

USA

Janina Kulka

Hungary

\author{
Kari Kuulasmaa \\ Finland \\ Belinda Lategan \\ Canada \\ Chia-Hwa Lee \\ Taiwan \\ Hannah Leslie \\ USA
}

Kent Lewandrowski

USA

Xu-Yong Lin

USA

Deqin Ma

USA

Pam Michelow

South Africa

Sergio Morini

Italy

Dwight Oliver

USA

\section{Giorgio Perino \\ USA}

Ari Probandari

Indonesia

Lakshmy Ramakrishnan India

Correspondence: elaine.zhang@biomedcentral.com

BMC Clinical Pathology, BioMed Central, 233 Spring Street, New York, NY

10013, USA

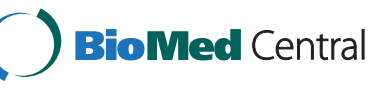


U.S. Rao

USA

Madhur Ravikumara

Australia

Massimo Rugge

Italy

Isabel Scaletsky

Brazil

Robert Schmidt

USA

Vivekanand Singh

USA
Steven Smith

USA

Neil Spooner

UK

Jerzy Stanek

USA

Simona Stolnicu

Romania

Ryan Sullivan

USA

Karoly Szuhai

Netherlands
Alexandar Tzankov

Switzerland

Nicola Volpi

Italy

Semir Vranic

Bosnia And Herzegovina

Michael Whitehouse

UK

Dehua Yu

China 